\title{
Investigating and promoting epistemological sophistication in quantum physics
}

\author{
Jessica R. Hoehn and Noah D. Finkelstein \\ Department of Physics, University of Colorado, 390 UCB, Boulder, CO 80309
}

\begin{abstract}
While the physics community has made significant strides to go beyond traditional conceptual and content mastery by focusing on student epistemologies, examinations of domain-specific epistemology have been less common in physics. This paper examines how students think of physics' connections to the real world in two different contexts: classical mechanics and quantum mechanics. We demonstrate domain-specific perspectives, show sophisticated reasoning by students (who hold different perspectives), and suggest that faculty perspective and instructional practices influence students' views.
\end{abstract}

\section{INTRODUCTION}

Students' attitudes, expectations, and epistemological beliefs (i.e. theory of knowledge) about physics not only influence how students learn but are an important goal of physics education itself [1-3]. However, as a research field, we have a tendency to treat physics as monolithic when measuring, assessing [4, 5], or talking about students' epistemologies. Although there are notable exceptions, including studies of students' epistemologies and expectations of experimental physics (e.g. [6]). In this study, we explore domain-specific epistemological commitments within physics, seeking to add to discussions around when and where we might treat physics as monolithic and where we consider sub-domains. We build off of our exploratory work which investigates splits in students' epistemologies in classical and quantum physics [7] on a set of six items from the Colorado Learning Attitudes about Science Survey (CLASS) [4], bifurcated to ask separately about classical and quantum physics. Here, we focus on one question central to our goals of physics teaching and examine the nature of the difference in students' beliefs about the relation of classical and quantum physics to the real world. We provide support for the following claims: 1) Students sometimes hold different epistemologies for classical and quantum physics; 2) Having different epistemological beliefs about classical and quantum physics can be a sophisticated stance, and we want students to be able to articulate this difference; 3 ) The item on relation of physics to the real world is valuable in sparking rich epistemological discussions and we investigate what these splits mean for students; 4) Instructor epistemological stance can contribute to students' epistemological development - it is important to be aware of your own domain-specific epistemologies and attend to them when teaching. By engaging with the rich discussions surrounding differences in students' classical and quantum epistemologies, we value moving away from a purely monolithic view of epistemology in physics.

\section{BACKGROUND}

Students' epistemological beliefs and attitudes about learning physics have been assessed and studied [8] with many surveys, e.g. the CLASS [4] and the Maryland Physics Expec- tations Survey (MPEX) [5]. Most published CLASS results come from studies of introductory physics courses in which the survey was given at the beginning and end of the course. The CLASS intentionally treats physics as monolithic, asking about students' beliefs and attitudes about physics as a whole. Traditional interpretation of CLASS results thus considers responses more consistent with expert views (as defined in original validation interviews [4]), or more favorable in introductory classical physics, as being more sophisticated. The results that we present here in which students report that quantum physics is less related than classical physics to the real world might be naively interpreted to mean students have less sophisticated or less expert-like views about quantum physics than they do about classical physics. While, in some instances, such an interpretation could be a legitimate conclusion, we instead turn to investigate further the domain specificity of students' epistemologies within physics. In the broader epistemologies research, there is growing consensus that students' epistemological beliefs are domain-specific [9, 10]. Usually, "domain" refers to academic discipline, but the possibility has been left open that the domain specificity could exist at a finer grain size (i.e. sub-disciplines of physics instead of physics vs. chemistry).

In a prior study [7], we administered a set of six CLASS items in Modern Physics courses, but asked students to respond twice, once asking about classical physics and once asking about quantum physics. We found significant splits between students' epistemological views of classical and quantum physics on some items, and we triangulated these quantitative results with interviews with some of the same students. We also found that, in some conditions and on some items, quantum physics instruction was associated with a prepost shift in students' responses to classical physics items. This preliminary study provided evidence of domain-specific epistemologies within physics, as well as raised questions about how to interpret and treat such split results. Rather than interpret the results to mean that students are less expertlike at quantum physics than they are at classical physics, the study suggests the possibility of interpreting students' reports of quantum physics being less intuitive and less tangibly related to real world experience as signals of sophistication.

Prior work in Modern Physics courses has shown that an instructor's interpretive stance, and how they attend to interpretation of quantum mechanics (QM) in the classroom, mat- 
ters for student learning $[11,12]$. Students in a class with faculty who held (and taught) from local-realist perspectives of quantum mechanics were more likely to take realist perspectives (e.g. the electron has a definite but unknown position in an atom). Students in a class taught from a quantum-matter view were far more likely to hold such views, and students in an "agnostic" perspective were likely to make their own interpretations, usually a classical-realist perspective. Along the same vein, we now argue that instructor epistemological stance also matters.

The present paper describes an extension of these two threads of work and further investigates the nature of these splits in students' epistemologies and the role of instructional positioning in our quantum physics classes.

\section{METHODS}

Course Context \& Participants: We administered a survey of epistemologies in classical and quantum physics in two courses at University of Colorado Boulder: Modern Physics (MP) and Quantum 1 (QM1). The MP course is the third semester in the introductory physics sequence, and serves as an introduction to QM. Resulting from several years of course transformations, the curriculum now emphasizes interactive engagement, conceptual foundations, and real-world applications of QM [13], and includes attention to physical interpretation of QM [12].

The QM1 course is the first semester of upper division QM, and this particular semester it was taught with a spins-first pedagogical approach (using McIntyre's textbook [14]). The course included clicker questions in lecture and had an optional one-credit co-seminar associated with it that approximately $25 \%$ of the class regularly attended. The content of the course had a focus on analytic problem-solving and preparation for continued advanced level study of QM. The instructors of these courses are both award winning instructors and have published on student learning in QM.

Students participated in this study as part of their course participation. There were 84 students from MP (68\% of class) who were typically 2nd year engineering majors, and 40 students from QM1 (93\% of class), typically junior physics or engineering physics majors. The MP course was approximately $12 \%$ female, QM1 was $37 \%$ female, and both courses enrolled predominantly White students.

Data Collection: We administered a survey in both the MP and QM classes, in two different semesters. The survey included the set of six bifurcated CLASS items from our preliminary study: items $6,23,28,35,40$, and 41 from the original instrument [4]. One block of questions was introduced by the instruction "For these questions, please think about your previous physics courses on motion, electrical phenomena, etc., which we are calling 'classical' physics in the items below," and the other was introduced by "For these questions, please think about past/upcoming courses on modern physics and/or quantum physics." The paired classical and quantum items were apart from one another on separate pages of the survey so as to disuade students from making direct comparisons. We note that the use of these questions is counter to the design and validation of the CLASS [4]; however, our goal was to determine if students held different commitments about classical and quantum physics. Students' comments in interviews from present and prior work [15] suggest that they interpreted "classical" and "quantum" the way we intended on the surveyed questions. The survey was given at the beginning and end of the MP course, but only at the end of the QM1 course.

In order to get a sense for the course contexts, we requested that the instructors of the courses also take the survey. They responded to the survey as they would personally answer the questions, and also reported for each quantum physics item the extent to which that particular topic was covered in their class. A free response section was also provided for the faculty to explain their reasoning. Finally, a review of course materials and syllabi demonstrated that the faculty addressed the issues of QM relation to the real world in class and that these curricula were consistent with the faculty stated perspectives.

At the end of the semester of the QM1 course, we conducted focus group interviews with $13(30 \%)$ of the QM1 students, in part for exploratory validation purposes. The students had taken the survey a week prior to the interviews, and as they worked in small groups we asked them to discuss how they responded to the bifurcated survey items and why. Together with the survey results, we used these student discussions to help us understand what the split epistemologies might mean for students learning QM.

Methods of Analysis: We focus our analysis on one particular item: The subject of [classical/quantum] physics has little relation to what I experience in the real world (item 35 in [4]). Although we ran both pre- and post-surveys in the MP class, we focus here only on the post administration because we are interested in looking at the classical/quantum splits at the end of the MP and QM1 classes, and not at pre/post shifts. We matched the data, and only included students in the item-level analysis who responded to both the classical and quantum version of a given item. The survey questions are given on a 5-point Likert scale from Strongly Disagree to Strongly Agree, but for analysis we collapse the results to a 3-point scale (Disagree, Neutral, Agree). We treat these data as ordinal, with three discrete categories rather than ordered points on a continuum, since we cannot assume the Neutral response to lie on a linear spectrum halfway between Disagree and Agree [4, 7]. In testing whether or not the classical/quantum splits were statistically significant, we use the Bhapkar test $[16,17]$ which uses information about how many students actually split their responses to determine if the overall distributions are different. We also test whether or not the distributions of responses on the quantum item are different between the two classes using a Mann-Whitney test.

In addition to the surveys and statistical tests, we examine student responses from the interviews about the question of 
interest, as well as the instructors' responses and reports of focus on this topic in class, to investigate what these epistemological splits mean for students, and how these may depend upon the instructional view and approach. This triangulation of data allows us to dive into how students are reasoning.

\section{RESULTS}

In both classes, there is a highly statistically significant split between students' classical and quantum responses $(p<$ 0.0001 in both cases). The majority of students disagreed with the statement for classical physics (Table I); that is, they think classical physics is related to their experiences in the real world. In both classes, far fewer students disagreed with the statement for quantum physics than they did for classical physics. Overall, students are less likely to report that quantum physics is related to their real-world experiences than classical physics. Although both classes had significant splits between classical and quantum physics, the quantum responses for the two courses look different from one another. Whereas the majority of MP students disagree with the quantum item, the distribution of responses for the QM1 students is bimodal. Collectively, the MP students think quantum physics is related to their real-world experience, but less strongly so than for classical physics. The QM1 students on the other hand are just as likely to disagree with the quantum statement as they are to agree. The two class distributions for the quantum version of the question are significantly different at the $\alpha=0.05$ level $(p=0.028)^{1}$.

TABLE I. Percentages of students who gave each possible combination of responses (Disagree, Neutral, Agree) on both versions of the item, The subject of [classical/quantum] physics has little relation to what I experience in the real world. Instructor responses are indicated by the shaded square.

\begin{tabular}{|c|c|c|c|c|c|c|}
\hline \multirow{2}{*}{$\begin{array}{l}\text { Modern Physics } \\
N=84\end{array}$} & & \multicolumn{5}{|c|}{ Quantum } \\
\hline & \multirow{5}{*}{ Classical } & & $\mathrm{D}$ & $\mathrm{N}$ & A & Total \\
\hline & & D & 51 & 17.9 & 13.1 & 82 \\
\hline & & $\mathrm{N}$ & 2.4 & 4.8 & 1.2 & 8.4 \\
\hline & & A & 2.4 & 2.4 & 4.8 & 9.6 \\
\hline & & Total & 55.8 & 25.1 & 19.1 & \\
\hline \multirow{6}{*}{$\begin{array}{l}\text { Quantum 1 } \\
N=40\end{array}$} & \multirow{6}{*}{ Classical } & \multicolumn{5}{|c|}{ Quantum } \\
\hline & & & $\mathrm{D}$ & $\mathrm{N}$ & $\mathrm{A}$ & Total \\
\hline & & $\mathrm{D}$ & 40 & 12.5 & 42.5 & 95 \\
\hline & & $\mathrm{N}$ & 0 & 0 & 2.5 & 2.5 \\
\hline & & A & 2.5 & 0 & 0 & 2.5 \\
\hline & & Total & 42.5 & 12.5 & 45 & \\
\hline
\end{tabular}

\footnotetext{
${ }^{1}$ While we have only included one item in this analysis, the remaining survey items showed varying degrees of splits (some with no split at all). This, in addition to the fact that the paired items were not presented next to each other on the survey, eliminates the possible interpretation that the split results are simply due to asking the same question twice.
}

Interviews with the QM1 students help to explain what this bimodal distribution or epistemological split means for the students. One group of four students was responding to the question about classical/quantum physics having little relation to their experiences in the real world, discussing what they answered for both classical and quantum versions, and why. Everyone responsed Disagree for classical, but the group was split between Disagree and Agree for the quantum version (Fernando and Diana responded Disagree, while Lucy and Stephanie responded Agree). Their conversation surrounding this question included the following exchanges:

Diana: ...I was thinking about... how the Sun wouldn't even be able to work if it wasn't for quantum tunneling, because you wouldn't get fusion...I was like, I experience the Sun every day, so [laughs].

Stephanie: ...I guess you're thinking about like details, and I was thinking more, big picture. Because I was thinking most of the things we interact with are too big to be like quantum.

...

Fernando: ...I guess one of the things I think about is like light and photons. Like that's all quantum.

Diana: Yeah, and we experience that every day too. Even if we're not aware that that's what we're experiencing.

Following this conversation, the group came to a consensus that as a collective group they would choose Neutral.

It is not surprising to see different results for the quantum item in the two classes, given that they are different student populations and different course contexts. However, the difference in the distributions that we do see is consistent with how the respective instructors answered the question themselves. The MP instructor answered Strongly Disagree, and throughout the course emphasized applications of quantum physics to students' worlds (quantum basis of color, the periodic table, and applications such as the laser, MRI, and nuclear energy/weapons). Furthermore, the course engages students in debating about the nature of how and when QM shapes their worlds. The QM1 instructor answered Neutral, with the following accompanying explanation: "Everything I experience arises in part from QM (light, material properties, everything) but nothing I experience is quantum mechanical, I have no direct experiences that are non-classical." The QM1 course engages in some discussion about the relevance of QM and its connections to other phenomena and branches of physics, but this was not a large portion of the class.

\section{DISCUSSION \& CONCLUSIONS}

In both the MP and QM1 classes, students almost unanimously agreed that classical physics is related to their realworld experiences, but were less likely to say the same for quantum physics. We consider the QM1 students' conversation about their differing views about classical and quan- 
tum physics to be indicative of epistemological sophistication. Fernando and Diana explain that they see quantum physics as related to their experiences because QM underlies things like nuclear fusion, light, and electronics (even though they might not be aware they are experiencing these things). Lucy and Stephanie explain that they see quantum physics as not being related to their experiences because everything they interact with is macroscopic (Lucy says she experiences turning on a TV every day, but not building one). This conversation is closely aligned with the QM1 instructor justification for answering Neutral. Ultimately, the selection of Neutral is a proxy for the instructor's own preference for a bimodal response (in some ways agree and in some ways disagree), which parallels both the course response overall and the focus group discussions. We consider being able to articulate these differences and engaging in this type of epistemological discussion to be sophisticated, and we aim for our physics students to be able to do these things.

We have seen that experts do not agree on the response to the quantum version of this question, and that the item is subject to different interpretations: real-world experience arises from versus is QM. We note that we have not validated these bifurcated questions from classical test theory, and as a result do not seek to use them to issue a survey to review all students' views about real-world connections of quantum and classical physics. Rather, we use these results to demonstrate that students can and do engage in sophisticated (and messy) reasoning about the connection of physics to the real world, and that they do have domain-specific perspectives. Such perspectives reflect the debates between the two course instructors and those in the broader community.

The distributions of responses to the quantum question from the MP and QM1 classes are significantly different from one another. Roughly $43 \%$ of QM1 students answered Disagree for classical physics and Agree for quantum physics, compared to only $13 \%$ of the MP students. This difference is consistent with the difference in instructor responses. At present, we cannot argue for causality, but we do argue that instructor stance and extent of emphasis in their teaching are contextual factors contributing to students' epistemologies.
We do not wish to suggest that one instructor stance is better or worse than the other, but we argue that the correlation between instructor stance and student responses implies that we should be aware of our own domain-specific epistemologies and attend to them when teaching. Furthermore, we note that whether or not we teach issues of interpretation or epistemological commitment, students will engage in such meaning-making [12]. One critique of this work could be that if professors' stances matter for students' epistemological development, then we should be looking for impact over time. We have pre and post data for the MP class and we do see pre to post shifts for some quantum items. However, existence of such shifts, or lack thereof, does not necessarily help us evaluate the impact of instructor stance because often we see regress on CLASS items over the course of a physics class [8], and thus the lack of a shift could be the result of instructor stance. Additionally, the pre survey was administered one week into the semester, at which point the MP instructor stance may have already been made clear to the students.

In this work, we wish to move away from adhering to universal epistemologies in physics, and allow space for domain specificity when we measure, assess, teach, and talk about students' epistemological commitments. The quantitative and qualitative results of this study demonstrate that students sometimes hold different beliefs about relation of classical and quantum physics to real-world experiences. We argue that these splits can be indicative of epistemological sophistication, and we call for more research that values and supports students in engaging in these types of rich epistemological discussions. Future work will examine the impacts of epistemological commitments on learning.

\section{ACKNOWLEDGMENTS}

The authors thank Benjamin W. Dreyfus, Andrew Elby, Ayush Gupta, and the anonymous students and instructors who participated in this study. Work supported by NSF TUES \#1322734, \#1323129, \#1548924 and a GRF.
[1] J.L. Docktor and J.P. Mestre, Phys. Rev. ST Phys. Educ. Res. 10, 020119 (2014).

[2] D. Hammer, Cogn. Instr. 12, 151 (1994).

[3] D. Hammer and A. Elby, J Learn. Sci 12, 53-90 (2003).

[4] W.K. Adams, et al., Phys. Rev. ST PER 2, 010101 (2006).

[5] E.F. Redish, J.M.Saul, and R.N. Steinberg, Am. J. Phys. 66, 212 (1998).

[6] B.R. Wilcox and H.J. Lewandowski, Phys. Rev. Phys. Educ. Res. 12, 010123 (2016).

[7] B.W. Dreyfus, et al., Phys. Rev. PER, In Review (2017).

[8] A. Madsen, S.B. McKagan, and E.C. Sayre. Phys. Rev. ST Phys. Educ. Res. 11, 010115 (2015).

[9] K.R. Muis, L.D. Bendixen, and F.C. Haerle, Educ. Psych. Rev.

$$
\text { 18, } 3 \text { (2006). }
$$

[10] J.A. Greene, W.A. Sandoval, and I. Bråten, Handbook of Epistemic Cognition, Routledge (2016).

[11] C. Baily and N.D. Finkelstein, Phys. Rev. ST Phys. Educ. Res. 6, 010101 (2010).

[12] C. Baily and N.D. Finkelstein, Phys. Rev. PER 11, 2 (2015).

[13] S.B. McKagan, K.K. Perkins, and C.E. Wieman, PERC Proc., AIP Press (2006).

[14] D.H. McIntyre, Quantum Mechanics, Addison-Wesley Educational Publishers Inc (2012).

[15] C. Baily, Ph.D. Thesis, University of Colorado Boulder (2011).

[16] X. Sun and Z. Yang, SAS Global Forum Proc., Texas (2008).

[17] V.P. Bhapkar, J. Am. Stat. Assoc. 61, 228 (1966). 